\title{
Coulomb crystallization in expanding laser-cooled neutral plasmas
}

\author{
T. Pohl, T. Pattard, and J.M. Rost \\ MPI for the Physics of Complex Systems, Nöthnitzer Str. 38, D-01187 Dresden, Germany
}

(Dated: October 31, 2018)

\begin{abstract}
We present long-time simulations of expanding ultracold neutral plasmas, including a full treatment of the strongly coupled ion dynamics. Thereby, the relaxation dynamics of the expanding laser-cooled plasma is studied, taking into account elastic as well as inelastic collisions. It is demonstrated that, depending on the initial conditions, the ionic component of the plasma may exhibit short-range order or even a superimposed long-range order resulting in concentric ion shells. In contrast to ionic plasmas confined in traps, the shell structures are built up from the center of the plasma cloud rather than from the periphery.
\end{abstract}

PACS numbers: $52.27 . \mathrm{Gr}, 32.80 . \mathrm{Pj}, 52.38 .-\mathrm{r}, 34.60 .+\mathrm{z}$

It is well known that, depending on the Coulomb coupling parameter $(\mathrm{CCP}) \Gamma=e^{2} / a k_{B} T$, a plasma may show long-range order, short-range order or no order at all. In general, ordering effects can be expected in the so-called strongly coupled regime $(\Gamma \gg 1)$ where the interparticle Coulomb interaction $e^{2} / a$ dominates the thermal energy $k_{B} T$ of the plasma particles. This parameter regime has been studied extensively in nonneutral plasmas of laser-cooled ions confined in ion traps $1,2,2,3,4,5]$. On the other hand, much less is known about the dynamics of finite, strongly coupled neutral plasmas without confinement. Due to their expansion, these plasmas are in a non-equilibrium state at all times, and it is not clear whether dramatic ordering effects such as Coulomb crystallization known from static trapped ionic plasmas can be observed in such a system.

Experimentally, cold neutral plasmas could be realized only recently by photoionizing a cloud of ultracold $(T \ll 1 \mathrm{~K})$ atoms [6, 7, 8]. Yet, under the present experimental conditions, the regime of a strongly correlated plasma cannot be reached [9, 10, 11]. Since the plasma is created in a completely uncorrelated state, the subsequent conversion of potential into kinetic energy rapidly heats both the electron and ion subsystem, suppressing the development of substantial correlations 9, 10]. Additionally, inelastic collisions with Rydberg atoms, previously formed by three-body recombination (TBR), and the TBR itself [1, 12] heat the electron gas. Therefore, $\Gamma_{i}$ decreases to unity while $\Gamma_{e}$ becomes even smaller, preventing strong correlation effects. However, further Doppler cooling of the ions during the plasma expansion has been suggested as a possible route to strongly coupled ultracold plasmas [13, 14].

In the following we provide the first theoretical description for the expansion of such a laser-cooled neutral plasma. As we will demonstrate, strong-coupling phenomena can indeed occur in such a system, leading to the formation of surprisingly differentiated patterns in the ionic plasma given the appropriate initial conditions. From a nonlinear dynamics point of view one might characterize these phenomena as self organization of a system in a non-equilibrium state.

In a first step we describe the collisionless plasma dynamics by a set of coupled Vlasov equations for the electrons and ions [15], neglecting any correlation effects. Cooling of the ions is modelled by introducing a FokkerPlanck term into the ion kinetic equation [16]

$$
\left(\frac{\partial f_{i}}{\partial t}\right)_{c}=\beta\left[\nabla_{\mathbf{v}}\left(\mathbf{v} f_{i}\right)+\frac{k_{B} T_{c}}{m_{i}} \Delta_{\mathbf{v}} f_{i}\right] .
$$

Here, $f_{i}$ is the one-particle distribution function of the ions, $m_{i}$ is the ion mass and the damping rate $\beta$ and the Doppler temperature $T_{c}$ are determined by the details of the laser cooling process. Assuming quasineutrality together with an adiabatic treatment of the electrons, one can show that the resulting ionic kinetic equation permits a Gaussian selfsimilar solution $f_{i}(\mathbf{r}, \mathbf{v}) \propto$ $\exp \left(-r^{2} / 2 \sigma^{2}-m_{i}(\mathbf{v}-\gamma \mathbf{r})^{2} / 2 k_{B} T_{i}\right)$, also found in the free plasma expansion problem [11, 15, 17]. The rmsradius $\sigma$ of the plasma cloud, the hydrodynamic velocity parameter $\gamma$ and the temperatures $T_{i}$ and $T_{e}$ evolve according to

$$
\begin{aligned}
\dot{\sigma} & =\gamma \sigma, \\
\dot{\gamma} & =\frac{k_{B} T_{e}+k_{B} T_{i}}{m_{i} \sigma^{2}}-\gamma(\gamma+\beta), \\
\dot{T}_{e} & =-2 \gamma T_{e}, \quad \dot{T}_{i}=-2 \gamma T_{i}-2 \beta\left(T_{i}-T_{c}\right) .
\end{aligned}
$$

As seen from Eq. (2), the action of the cooling laser is twofold. The ion temperature is driven towards its equilibrium value $T_{c}$, while $\gamma$ is linearly damped out on a timescale of $\beta^{-1}$. Eq. (2) still has one integral of motion, $\sigma^{2} T_{e}=$ const., reflecting the adiabatic electron cooling during the plasma expansion. If $T_{i} \ll T_{e}$, an asymptotic description of the plasma dynamics can be obtained by neglecting terms of order $\gamma$ compared to the damping rate $\beta$ in Eq. (2), which yields in the long-time limit

$$
\sigma^{2} \approx \sigma^{2}(0) \sqrt{1+2 \frac{k_{B} T_{e}(0)}{\beta m_{i} \sigma^{2}(0)}} t \quad \stackrel{t \rightarrow \infty}{\propto} \sqrt{t} .
$$

This is in marked contrast to the free expansion, where $\sigma^{2} \approx \sigma^{2}(0)+\frac{k_{B} T_{e}(0)}{m_{i}} t^{2} \stackrel{t \rightarrow \infty}{\propto} t^{2}$. Therefore, continued 


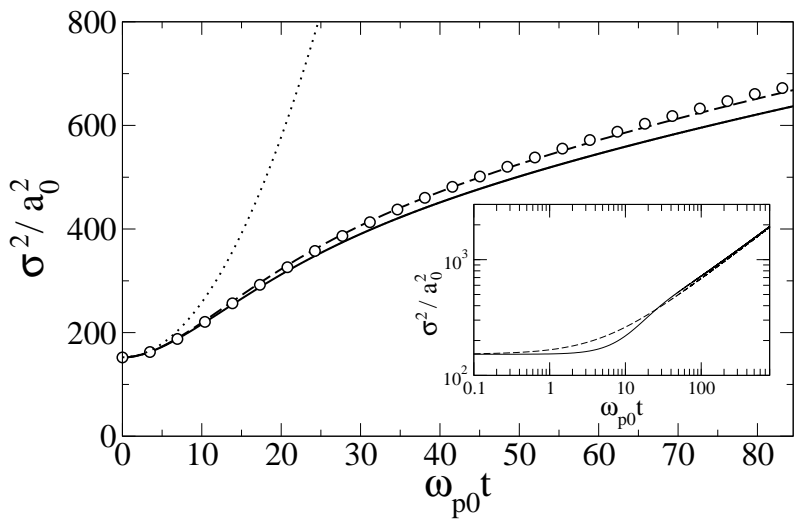

FIG. 1: The size of a laser-cooled plasma as compared to that of a freely expanding plasma of the same initial-state parameters (dotted). The solution of Eq. (2) (dashed) matches the PIC-treatment (circles), showing that the difference to the hybrid-MD simulation (solid) comes from the ionic correlations. The inset shows a comparison of the numerical solution with the analytical approximation Eq. (3).

Doppler cooling of the ions not only reduces their temperature but also drastically retards the decrease in plasma density, supporting development of strong correlations.

In a second step, we have performed a more elaborate numerical simulation based on a hybrid method treating the two plasma components on different levels of sophistication. Since the electrons are not strongly coupled and their relaxation time, determined by the electron plasma frequency, is small compared to both the plasma expansion time and the inverse of the ionic plasma frequency $\omega_{p}=\sqrt{4 \pi e^{2} \rho / m_{i}}$, an equilibrium fluid model provides an adequate description of the electron dynamics [1]. We account for the initial electron evaporation by determining the fraction of trapped electrons from the results of Ref. [6]. The ions move as classical particles under the action of the electronic mean field and the direct ion-ion interaction calculated with a particle tree-procedure developed in [18]. In analogy to Eq. (1), cooling is described by adding a Langevin force to the ion equations of motion. The electron temperature is determined by energy conservation for the total system consisting of the plasma and the radiation field. This hybrid treatment allows us to study effects of strong ion correlation over long times, since atomic timescales need not be resolved as in a full molecular dynamics (MD) simulation [9, 19].

In Fig. 1) we compare the time dependence of the plasma rms-radius obtained from Eq. (2) with the hybridMD simulation and the analytical approximation Eq. (3). There is good overall agreement between the two numerical approaches. Moreover, they both nicely reproduce the asymptotic $\sqrt{t}$-behavior of Eq. (3). On the other hand, the width calculated from the MD simulation is significantly shifted to lower values. A comparison with a particle-in-cell (PIC) treatment of the ions, also shown in Fig. 11 clearly reveals that the slower plasma expansion is due to the negative correlation pressure [9, 17], which partly compensates the thermal electron pressure. Note that here the influence of ion correlations is completely different from the case of free plasma expansion, where the initial correlation heating was found to dominate the negative correlation pressure and hence accelerates the plasma expansion [17].

Up to this point, we have taken into account the electron-ion interaction on the basis of a mean field description only. However, it has been found that electronion collisions leading to the formation of Rydberg atoms through TBR may significantly alter the expansion dynamics at these low electron temperatures [6, 11]. In order to include these processes in our description, we use a Monte-Carlo treatment 12, 20] to account for TBR and inelastic electron-Rydberg atom collisions. In addition to these processes the influence of the cooling laser on the Rydberg atoms should be addressed. By the very nature of the cooling process, a significant fraction of the ions is found in an excited state at all times. Thus, TBR may produce doubly-excited, and hence autoionizing, Rydberg atoms with a considerably large rate. (This process is in close analogy to the production of autoionizing Rydberg states by "isolated-core excitation" [21].) For low enough principal quantum numbers, the autoionization rate of these states becomes comparable to and even exceeds the radiative decay rate of the excited core. For the case of strontium, the l-averaged autoionization rate becomes important at $n \approx 50$. For electron temperatures of the present type of experiments, Rydberg atoms typically recombine into states with much higher $n$ so that Auger ionization does not play a role initially. However, in the course of the gas evolution the Rydberg electron moves down the energy ladder by subsequent inelastic electron-atom collisions. Since the energy shift of the core transition used for laser cooling is of the same order of magnitude as the autoionization rate [25], the Rydberg atoms formed by TBR are still resonant with the cooling laser. Hence, even if the timescale of collisional deexcitation is longer than the lifetime of the coreexcited state, the cooling laser will continue to drive the core transition so that the atom will be in the core-excited state for a significant fraction of time. Because the energy connected with the core transition is of the order of $10^{4} \mathrm{~K}$, each free electron produced by autoionization will rapidly leave the plasma volume. Hence, the combined action of the cooling laser, TBR and collisional deexcitation is expected to remove electrons from the plasma and destroy the plasma, until recombination stops when the electron density has become too small. In order to suppress this electron loss one has to choose initial conditions which lead to a tolerable TBR rate 22 .

If time and length are measured in units of the initial ion plasma frequency $\omega_{p 0}$ and Wigner-Seitz radius $a_{0}$, respectively, the initial plasma state is characterized by 


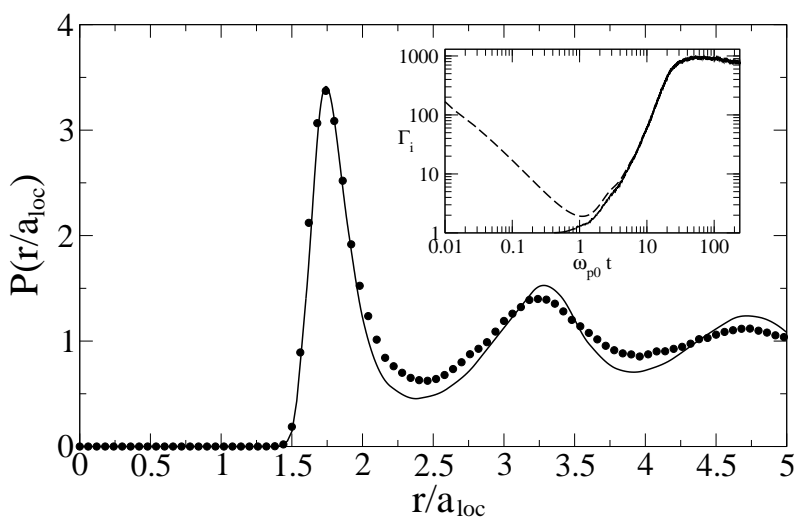

FIG. 2: Distribution of scaled inter-ionic distances after a time of $t=52 \mu \mathrm{s}\left(\omega_{p 0} t=240\right)$ compared to the calculated pair-correlation function of an OCP at $\Gamma_{i}=700$ [24]. The inset shows the ionic CCP calculated by different methods (see text for details).

five parameters: the number of ions $N_{i}$, the initial electron and ion CCPs, the value of $\Gamma_{c}$ corresponding to the Doppler temperature $T_{c}$, and the ratio of the cooling rate to initial ionic plasma frequency $\omega_{p 0}$. The exact values of $T_{c}$ and $T_{i}(0)$ are not important for the plasma expansion dynamics, since both are negligible compared to the electron temperature. According to Eq. (2), for fixed $\beta$ the time evolution of the scaled density only depends on the product of $\Gamma_{e}$ and $N_{i}^{2 / 3}$ (if $T_{i} \ll T_{e}$ is neglected in the second equation of Eq. (2)), the expansion being slower if $\Gamma_{e} N_{i}^{2 / 3}$ becomes larger. Hence, in order to slow down the plasma expansion sufficiently for spatial correlations to develop, one may prefer to increase the ion number since reduction of the electron temperature becomes ultimately incompatible with the objective of limiting TBR. A further constraint on the initial conditions arises from the fact that $\Gamma_{i} \approx 10^{3}$ in order to observe ordering effects. Typically, ion temperatures of the order of $1 \mathrm{mK}$ can be achieved through Doppler cooling, meaning that the initial ion density must be at least about $10^{8}$ $\mathrm{cm}^{-3}$. Finally, the cooling rate $\beta$ must be of the order of $10^{-1} \omega_{p 0}$ to sufficiently slow down the plasma expansion that correlations can develop. Since $\beta\left(\propto 1 / m_{i}\right)$ decreases faster than $\omega_{p 0}\left(\propto 1 / \sqrt{m_{i}}\right)$ with increasing ion mass, it is advantageous to consider relatively light ions, for which sufficiently high cooling rates can be obtained experimentally. We therefore choose Be ions for our simulations, for which laser-cooling has been experimentally demonstrated earlier in nonneutral plasmas 23.

The most striking result of our simulations is a latticetype crystallization of the ions or even their arrangement in concentric shells if the plasma expansion is slow enough. The emergence of such an order depends sensitively on the initial conditions. We have found a latticetype crystallization in the expansion of a plasma of 20000 Be-ions with an initial density of $1.1 \cdot 10^{8} \mathrm{~cm}^{-3}$ and an electron temperature of $T_{e}=29 \mathrm{~K}$, cooled with a damping rate of $\beta=0.15 \omega_{p 0}$ to an ionic temperature of $T_{c}=2$ $\mathrm{mK}$. The value of $\Gamma_{i}=750$ after $52 \mu \mathrm{s}$, simply calculated from the ion temperature and the average Wigner-Seitz radius, suggests strong ordering of the ionic component. However, as pointed out in [9] the CCP calculated in this way may have no meaning as a measure of correlations, since the expanding plasma does not reach a global equilibrium. A more reliable quantity, namely the distribution of inter-ionic distances, is shown in Fig. 2. In order to account for the nonhomogeneity of the plasma we have scaled the inter-particle spacing by the WignerSeitz radius $a_{l o c}$ determined by the local density between the corresponding particles. For comparison, the paircorrelation function obtained from the HNC-equations of a homogeneous one-component plasma 24 is also shown. (However, the distribution function shown in Fig. 2 should not be understood as a pair-correlation function in a strict sense, since in the present case the plasma is neither isotropic nor homogeneous.) The remarkable agreement shows that under present conditions the calculated CCP indeed indicates the degree of order in the expanding plasma and that the system has reached a state of local equilibrium far beyond the known crystallization limit of $\Gamma_{i} \approx 174[4]$. In order to study the dynamics of the crystallization process we have determined $\Gamma_{i}$ also from the numerically obtained average correlation energy together with an analytical approximation for this quantity [17]. A comparison of the CCPs calculated by both methods is shown in the inset of Fig. 2 Initially there are large deviations between both calculations reflecting the nonequilibrium character of the early plasma state. However, after some inverse plasma frequencies, the ion system reaches a local equilibrium and the CCPs obtained from the different methods become identical. At longer times both curves may diverge again due to a freezing out of ordered structures when the density be-

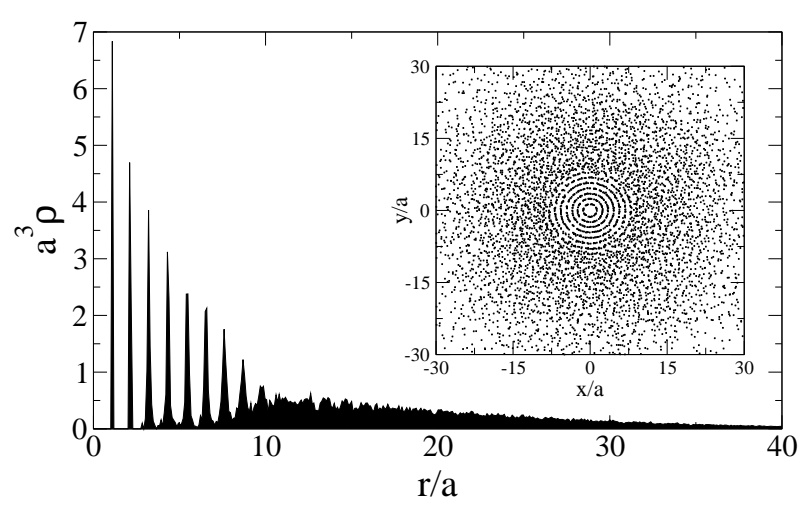

FIG. 3: Radial density after a time of $t=24 \mu \mathrm{s}\left(\omega_{p 0} t=\right.$ 110). The inset shows a two-dimensional cut through the plasma cloud, clearly revealing the formation of concentric shells. (For better contrast, cuts with $x=0, y=0$ and $z=0$, respectively, have been overlayed.) 


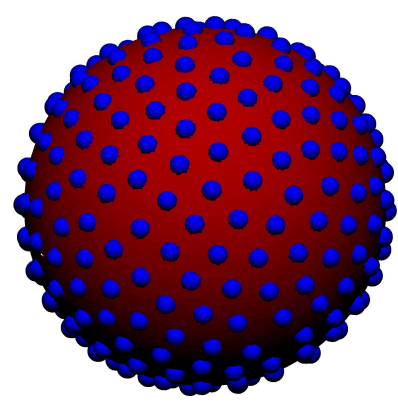

FIG. 4: (Color online) The $5^{\text {th }}$ shell of Fig. 3 demonstrating significant intra-shell ordering.

comes too small.

If we further slow down the plasma expansion, by increasing the product $\Gamma_{e} N_{i}^{2 / 3}$, the system exhibits formation of concentric shells rather than relaxation into lattice-type structures. This is demonstrated in Fig. 3 where the radial ion density is shown at $t=24 \mu \mathrm{s}$ for a plasma with $\Gamma_{e}=0.15$ and $N_{i}=50000$ while all other parameters equal those used for Fig. 2 Besides this radial ordering into concentric shells, there are strong intra-shell correlations, also found in trapped nonneutral plasmas 2]. In Fig. 4] we show one of the shells formed in the simulation of Fig. [3] The development of a hexagonal-lattice like ordering is evident, which is however considerably disturbed by the curvature of the shell. A closer look on the emergence of the order shows that in the early stages of the plasma evolution a cubic-lattice like structure is formed. However, after some ten inverse plasma frequencies the ions rearrange to form concentric shells starting from the plasma center, where the density is highest, in contrast to trapped nonneutral plasmas where the shell formation was observed to proceed from the periphery to the center of the cloud [5]. If the expansion is faster, as in the first example (Fig. 2), ionion collisions are less frequent and after the initial phase of local ordering the density is already too low for the rearrangement into shells, such that the lattice structure survives during the expansion.

In summary, we have followed the long-time dynamics of laser-cooled, expanding ultracold plasmas on the basis of a hybrid-MD simulation, allowing for a full treatment of the strongly coupled ion dynamics. The results show that cooling during the plasma expansion drastically modifies the expansion dynamics leading to an exotic type of plasma where the electron component is weakly coupled while the ion component shows strong coupling effects which manifest themselves in the development of lattice-like structures (short-range order) or even the formation of concentric shells (long-range order) depending on the expansion dynamics. Interesting questions concerning this novel system, like the behavior of ion collective modes or the influence of different density profiles on the details of the structure formation process, have to be addressed in future studies.

Financial support from the DFG through grant RO1157/4 is gratefully acknowledged.

[1] A. Rahman and J.P. Schiffer, Phys. Rev. Lett. 57, 1133 (1986).

[2] D.H.E. Dubin and T.M. O'Neil, Phys. Rev. Lett. 60, 511 (1988).

[3] S.L. Gilbert, J.J. Bollinger, and D.J. Wineland, Phys. Rev. Lett. 60, 2022 (1988).

[4] D.H.E. Dubin and T.M. O'Neil, Rev. Mod. Phys. 71, 87 (1999).

[5] H. Totsuji, T. Kishimoto, C. Totsuji, and K. Tsuruta, Phys. Rev. Lett. 88, 125002 (2002).

[6] T.C. Killian, S. Kulin, S.D. Bergeson, L.A. Orozco, C. Orzel, and S.L. Rolston, Phys. Rev. Lett. 83, 4776 (1999).

[7] S. Kulin, T.C. Killian, S.D. Bergeson, and S.L. Rolston, Phys. Rev. Lett. 85, 318 (2000).

[8] T.C. Killian, M.J. Lim, S. Kulin, R. Dumke, S.D. Bergeson, and S.L. Rolston, Phys. Rev. Lett. 86, 3759 (2001).

[9] S.G. Kuzmin and T.M. O'Neil, Phys. Rev. Lett. 88, 065003 (2002).

[10] M.S. Murillo, Phys. Rev. Lett. 87, 115003 (2001).

[11] F. Robicheaux and J.D. Hanson, Phys. Rev. Lett. 88, 055002 (2002).

[12] F. Robicheaux and J.D. Hanson, Phys. Plasmas 10, 2217 (2003).

[13] S.G. Kuzmin and T.M. O’Neil, Phys. Plasmas 9, 3743 (2002).

[14] T.C. Killian, V.S. Ashoka, P. Gupta, S. Laha, S.B. Nagel, C.E. Simien, S. Kulin, S.L. Rolston, and S.D. Bergeson, J. Phys. A 36, 6077 (2003).

[15] D.S. Dorozhkina and V.E. Semenov, Phys. Rev. Lett. 81, 2691 (1998).

[16] H.J. Metcalf and P. van der Straten, Laser Cooling and Trapping (Springer, New York, 1999).

[17] T. Pohl, T. Pattard, and J.M. Rost, Phys. Rev. A 68, 010703(R) (2003).

[18] J. Barnes and P. Hut, Nature 324, 446 (1986).

[19] S. Mazevet, L.A. Collins, and J.D. Kress, Phys. Rev. Lett. 88, 055001 (2002).

[20] C.K. Birdsall, IEEE Trans. Plasma Sci. 19, 65 (1991).

[21] T.F. Gallagher, Rydberg Atoms (Cambridge University Press, 1994).

[22] P. Mansbach and J. Keck, Phys. Rev. 181, 275 (1969).

[23] L.R. Brewer, J.D. Prestage, J.J. Bollinger, W.M. Itano, D.J. Larson, and D.J. Wineland, Phys. Rev. A 38, 859 (1988).

[24] K.C. Ng, J. Chem. Phys. 61, 2680 (1974).

[25] For the specific case of strontium, the energy shift and the autoionization rate were found to be almost identical. 